\title{
PERCEPCIÓN, IDENTIFICACIÓN Y REFLEXIÓN DE LA HISTORIOGRAFÍA DE LAS ÚLTIMAS DÉCADAS DEL SIGLO XX EN EL PERÚ
}

\author{
Carlos DE LA CRUZ VILLANUEVA \\ Universidad Ricardo Palma \\ carlos.delacruz@urp.edu.pe
}

"Fermin y Corina, experiencias y enseñanza de vida.

Muchas gracias abuelos."

\begin{abstract}
"Canela y Calíope, llenas de curiosidad, construyen un lugar en el mar de tinieblas que la vida desarrolla, no se imaginan cuánto me enseñan.

Gracias pequeñas hijas"
\end{abstract}

\section{RESUMEN}

Las siguientes líneas tienen como objetivo ingresar, aunque en forma muy dispersa, a la comprensión del desarrollo de la historiografía peruana de las últimas décadas del siglo XX, mediante el análisis de algunos textos que han abordado el tema. Esta observación preliminar busca identificar algunos elementos, indicadores y apreciaciones a la labor del historiador que cumple la función y crea condiciones que hacen posible su profesión. Ello a través del análisis o balances historiográficos, entrevistas y opiniones que hacen visualizar dichas condiciones.

\section{PALABRAS CLAVE}

Historiografía, el quehacer histórico en el Perú, balances historiográficos, historiador en el Perú

\section{PERCEPTION, IDENTIFICATION AND REFLECTION OF THE HISTORIOGRAPHY OF THE LAST DECADES OF THE 2OTH CENTURY IN PERU}

\begin{abstract}
The following lines have as aim to enter, although in a very dispersed way, the understanding of the development of the Peruvian historiography of the last decades of the twentieth century, through the analysis of some texts that have approached the subject. This preliminary observation seeks to identify some elements, indicators and appreciations to the work of the historian who fulfill the function of conditions make their profession possible, through analysis to historiographical balances, interviews and opinions that make to visualize these conditions.
\end{abstract}

\section{KEYWORDS}

Historiography, what to do historical in Peru, historiographic balances, historian in Peru 


\section{Ideas preliminares}

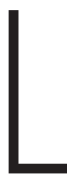

as siguientes líneas son el inicio de un trabajo mayúsculo, que con los años espero se siga fortaleciendo, son a su vez una percepción preliminar del desarrollo de la labor del historiador peruano. Esta mirada surge por comprender el hecho del lento, positivo y progresivo crecimiento de la presencia de la labor histórica en nuestro país en el siglo XXI. Ello se evidencia en el contexto de las últimas dos décadas en las cuales nuestro país ha vivido un crecimiento y contracciones económicas, que en líneas generales han impulsado esta presencia y que se manifiestan a través de mayor presencia de convenios internacionales de investigación, financiamiento, fondos contravalor, becas, crecimiento de instituciones universitarias, mayor presencia de estudios generales y/o básicos en las universidades públicas y privadas, renovaciones de planas docentes en las universidades, múltiples generaciones trabajando en conjunto, el estímulo de maestros universitarios, exigencia y requerimientos profesionales, la competencia profesional, el desempeño profesional, entre otras.

A esto podemos agregar que la presencia de coyunturas y problemáticas de interés nacional e internacional han estimulado una mayor reflexión y apertura intelectual. También un catalizador intelectual, muy interesante y necesario, las actividades académicas con respecto al Bicentenario de la Independencia, lo cual está generando un gran impulso por parte de diversas instituciones por mejorar el alcance y la renovación intelectual referidos a dicho acontecimiento y magno hecho histórico de nuestro presente, pasado y futuro de la historia del Perú. Tenemos que agregar el gran impulso por parte de jóvenes intelectuales de diversos sectores socioeconómicos que se han integrado a la labor de historiadores que en nuestro país a pesar de las limitaciones están marcando una presencia en diversos ámbitos del quehacer social, político y económico. Este trabajo esbozará una breve reflexión orientada a comprender cuál es la percepción de la historiografía peruana en las últimas décadas del siglo XX, lo cual para el interés particular será evaluado como una condición al desarrollo de la historiografía del siglo XXI.

\section{Identificando antecedentes}

La sociedad se desarrolla en el espacio que ocupa a través del tiempo, constituyendo, comprendiendo y adaptándose a múltiples situaciones, acontecimientos y hechos que son la expresión social de su presencia en el planeta. Pero la existencia de esos pasos o huellas sociales en el tiempo son materializadas gracias al trabajo del historiador, aquel personaje, que a través de su observación, análisis, descripción, interpretación y síntesis logra resaltar la conformación histórica de la sociedad en el devenir.

Esta loable y complicada labor profesional es en nuestro país desde hace muchas décadas, limitada, las razones son diversas; desde la falta de interés social y político, inversión y financiamiento en proyectos de desarrollo, diversas situaciones laborales de los historiadores, entre otras. A pesar que en el siglo XXI se evidencia un exponencial incremento, no existe una idea de proyecto de consolidación permanente como parte de necesidad del Estado y en caso de lo privado los intereses son mediáticos. Esta situación evidencia, al parecer, una suerte de continuidad, que reflexionando estriba en una serie de situaciones; 
como la falta de un proyecto nacional y privado de desarrollo de investigación y difusión histórica constante', la falta de un proyecto en conjunto por parte de los historiadores e incluso el hecho de no estar organizados institucionalmente vienen siendo hoy en día el pretexto que enarbolan ciertas agrupaciones para el desarrollo histórico en el Perúr.

Nuestra reflexión nos lleva a pensar ¿de qué depende el quehacer histórico en el Perú? Una respuesta, es que depende de múltiples factores. Pero, si nos concentramos en uno veremos todo un universo complejo, opinemos sobre quién hace la historia, que en este caso es el historiador. Y sobre ello ya algunos historiadores han opinado al respecto, en estas líneas trataré de condensar algunas opiniones de estos historiadores de las últimas décadas del siglo XX que han podido plasmar a través de balances, memorias, entrevistas u opiniones.

En principio, la labor de un historiador está compuesta de múltiples dimensiones que son parte de su ejercicio profesional. Estas son su ámbito personal, en el cual se incluye su formación familiar y su experiencia de vida. La segunda es la influencia que ejerce en el entorno socio económico en el cual se forma y desarrolla una serie de alcances o limitaciones en el tiempo. La tercera es el contexto y las coyunturas que afronta en su itinerario de vida. Otras dimensiones que influyen y que son necesarias mencionar son: la institución universitaria de formación, las redes sociales de las cuales forme parte 0 en las cuales se pueda haber constituido en el tiempo, una dimensión también influyente son los estudios académicos de formación intelectual en el extranjero, becas o premios.

Todas estas dimensiones son parte influyente en la profesionalización del quehacer histórico, además debemos mencionar que el historiador se entrega a un ser social del cual deviene y en el cual versa sus relatos que están nutridos de metodológicas y científicas formas que validan la opinión, transformándola en información veraz. Además, su prosa evidencia una necesidad reflexiva, pues está influenciada por las motivaciones filosóficas, sociales y políticas, fieles reflejos de su tiempo o rememoración de otros momentos. Por último, es innegable que el historiador es poseedor de una vena literaria que motiva, inspira $u$ apasiona y que es reflejo de su grado de entrega a su profesión. Todo lo anteriormente mencionado nos lleva a una idea, el historiador pueda que componga una investigación social o un conocimiento científico o posiblemente un discurso, tal vez una descripción empírica validada metodológicamente, pero de lo que sí estamos seguros es que su producto es gracias a un análisis complejo de una variedad de fuentes, generando un discurso que se convertirá en un testimonio veraz que vincula a los individuos en el tiempo y que se transforma en el devenir.

Al visualizar a los historiadores en el Perú en las últimas décadas del siglo XX, podemos apreciar que se desempeñan en diversas áreas o lugares donde al parecer no todos realizan

1 Sin negar la labor que en forma continua se realiza por parte de instituciones universitarias, instituciones como el IEP y otras que en forma esporádica realizan esfuerzos conjuntos en pro del desarrollo de la historia en el Perú, fundaciones privadas y en ocasiones en fondo editorial del BCRP.

2 Con respecto a la polémica véase http://larepublica.pe/cultural/888158-polemica-por-la-creacion-delcolegio-de-historiadores-del-peru. En cuanto al crecimiento y difusión de esta polémica en redes sociales ha sido motivo de una discusión por diversas generaciones, evidenciando opiniones dispersas, encontradas, algunas airadas y con algún trazo coherente académico corporativo. En lo particular considero necesario una organización institucional que se debe nutrir con el tiempo en su construcción, teniendo como base un proyecto histórico para el Perú, que sea más allá de un aseguramiento de empleo para el historiador. 
su labor como historiadores, lo cual es cuestionable, pero es parte de su vida profesional u al menos ha ganado ese espacio. Pues sí partimos del pensamiento de que el historiador debe escribir historia y se dedica a investigar a tiempo completo, lo cual es una situación poco frecuente en el Perú, pero existente. Nos deja con un dilema, si no es investigador a tiempo completo ¿a qué se dedica?

En 1999 Miguel Glave trató de puntualizar que la actividad en historia en nuestro país se ha ido nutriendo de lo avanzado por los historiadores peruanos, el contexto nacional e internacional, y los intercambios entre investigadores de otras nacionalidades que han escrito sobre el Perú, los llamados peruanistas. Él ve con optimismo a pesar de no evidenciar una esencia en común en los historiadores de que existe un afán sea individual o de agrupaciones por escribir historia, replanteando nuevos tópicos, y dando síntesis a otros temas y porqué no mencionarlo dejando en el pasado otros. Pero un punto que resalta es la utilidad social del historiador que en las décadas en mención vive a la deriva por la utilidad de sus conocimientos para la sociedad, no solo buscando un espacio socio profesional, una remuneración para el sustento personal, familiar y profesional que gravita a la espera de un gran equilibro en el crecimiento económico de aquellos años.

En las décadas en mención su desempeño, en el mejor de los casos, será el idóneo y si no lo es, su labor será la de docente universitario o en alguna otra institución educativa, como Academias, Institutos y colegios, siendo profesor de algún curso de historia o derivado de ciencias sociales; en otros casos se desempeñará en algún archivo histórico o de gestión, en bibliotecas, analista de gestión, algún trabajo burocrático, o administrativo, por ultimo podríamos agregar que este personaje podría desempeñarse en diversos oficios o actividades completamente desligadas de la labor histórica.

Carlos Lazo (2005) aprecia en el historiador peruano una problemática y en forma mucho más puntual y conceptual enmarca al intelectual de acuerdo a la forma de historia que realiza, es decir historia social, económica, ideológica y política. Y como el contexto y el entorno lo va formando al historiador, Lazo clasifica al historiador bajo los parámetros interesantes, pues los va a agrupar en relación a su grupo social de origen y por el cual van a escribir la historia, condicionada por su ser social; los clasifica en tipos de Historiador: historiadores señores, historiadores clientes, historiadores empresarios, historiadores revolucionarios. Una postura muy dura y que toma años de experiencia comprender y que es interesante, pues coloca al intelectual como un ente que responde a su sociedad partiendo de su ser social al cual pertenece.

Al parecer, la relación problemática de finales de los 90, nos permite visualizar momentos o indicadores críticos de los historiadores, que pueden ser similares unas décadas antes; otros lapsos coyunturales similares nos evidencian en opinión de Macera en 1968 un profesional a la defensiva, el gran historiador crítico comprende que el historiador peruano desarrolla, preocupado por la validez científica y su opción y participación político social, plantea la problemática de la historia a partir de la premisa que el historiador es un personaje a la defensiva con una latente inseguridad de la utilidad de su oficio; lo interesante es que clasifica a los historiadores bajo contextos que van a ser el marco en donde se desarrolla la intelectualidad referida a la historia. 
A su vez Macera utiliza un criterio metodológico para agrupar a los historiadores en base a lo que él bautiza como generaciones ${ }^{3}$ y están constituidas de acuerdo a criterios en donde la universidad intervino o fue afectada directamente por el desarrollo en que afrontó la sociedad peruana desde 1900 hasta fines de los 60 del siglo XX; interesante planteamiento, pues nos sugiere un acercamiento al intelectual desde su centro de procreación el cual es la Universidad San Marcos, eje central de la intelectualidad en el Perú. Dicha sugerencia es apropiada para fines de la investigación del profesional, pues sirve para el entendimiento de este a partir del contexto inmediato y nos informa que la universidad y la sociedad estaban íntimamente relacionadas, es decir el estudiante universitario íntimamente relacionado con la situación de su sociedad en el tiempo y como respondía a la misma con intervenciones directas plasmadas en la producción de un colectivo cohesionado consciente o inconscientemente, el historiador en su discurso daba respuestas a las inquietudes sociales de su tiempo.

Otro punto interesante de esta apreciación generacional, es que a pesar de no desarrollar un programa histórico a largo plazo, las generaciones se interconectan mostrando el hecho de que esas generaciones no son solo de efecto aislado para su propio tiempo; es decir sus alcances y limitaciones nos fueron heredadas y heredamos a través del tiempo, cada historiador y sus obras son eslabones de una misma cadena y gracias ello la historia logró y logra ganar espacio, pero también formó un carácter de inseguridad en su discurso.

Un aspecto central de esta apreciación es que el historiador entre los 60 y 90 ha logrado una labor histórica intensa realizando trabajos sobre la conquista, el virreinato y la república, mostrándonos problemas de la historiografía y renovando el conocimiento. En este sentido esta herencia sin proyectos a futuro, nos muestra una historia renovadora y que debería ser contemporánea y fiel muestra de que los historiadores que viven en un contexto determinado y responden a él en la medida de sus posibilidades, pero el comprender la evolución intelectual de los historiadores es ingresar a la formación intelectual profesional como la UNMSM; los problemas o proyectos inconclusos heredados han dado al historiador metodología, forma, estilo, pero al parecer no lo han dotado de la capacidad de hacer útil su labor y hacerlo interventor en plantear una suerte de solución o recomendaciones, por medio de un plan.

El historiador si quiere lograr una gran renovación debe tener un plan; debe escribir una historia sobre el presente, es decir una historia que conecte en forma retrospectiva el pasado y el presente nos ayudaría a construir el futuro, ello nos daría una presencia y utilidad y nuestro oficio no estaría recluido a la mera descripción del pasado ya que posee la conexión generacional y esa reacción vital de escribir por las coyunturas presentes rastreando orígenes a los problemas sociales lograr una historia del presente que es de vital importancia, que recuperara la necesidad social, pues la historia es vista como solo una relación de hechos pasados, con síntesis interesantes y reflexiones plausibles, pero que no pueden conectar el pasado con el presente, coincidir en los esfuerzos, una reorientación temática total de cuestiones más próximas compatibles a la actual de la historia de adelante

3 Menciona las generaciones, como la del novecientos, la de la reforma universitaria, la de la clausura y una tercera que se le podría atribuir el hecho de la inserción de los nuevos grupos sociales. 
hacia atrás y en retrospectiva, pero lo más importante es tener despejada la conciencia de su situación histórica.

Flores Galindo (1988) hace hincapié en que la historiografía en el Perú gravita en relación a la actitud del historiador y sus propias necesidades, su quehacer es fiel reflejo de su entorno y tiempo que le tocó vivir y lo realiza a través de las instituciones de las que él es parte, me refiero a la historia hecha por los institutos como el IEP y los investigadores extranjeros que realizan historia sobre el Perú, los llamados peruanistas, quienes influencian y han desarrollado en parte la historia de múltiple enfoque e interconectada con otras ciencias tal como Macera apreciaba que la reunificación de los esfuerzos era a partir de hacer una labor en conjunto abriendo puentes con las ciencias sociales y superar el presentismo ${ }^{4}$. Según Flores Galindo el historiador en las instituciones, en los institutos o centros especializados logra una mejor integración intelectual con ideas que llegan desde fuera que renuevan y constituyen las nuevas visiones de la historia, es decir el desarrollo de las temáticas intelectuales obligadas por las coyunturas del tiempo que le toca vivir al historiador; lo interesante es que formula una opinión en donde la historia es hecha a la imagen de sus historiadores, lo cual es algo positivo pero tiene un problema, pues ello nos explica que a pesar de la recomendación hecha por Macera en el año de 1968 no se tomó en cuenta el plan, sino la influencia articulada y agrupada como solución para realizar la historia y realizar un deslinde intelectual.

Nuevamente a mediados de los noventa Quiroz Chueca (1995) realiza un balance no solo en el aspecto temporal, espacial y contextual, sino también a los representantes de este tipo de historiografía, la llamada Dependentista, tomando como tópico un punto en el cual los historiadores en el Perú han escrito sobre la independencia y el desligue del orden virreinal desde las posturas más patriotas hasta las multifactoriales que han dado como fruto una renovación a través de la redirección copiosa en si de un hecho histórico, en donde esta historiografía encamina todos sus esfuerzos por explicarnos la situación de atraso y oportunidades perdidas, juzgando al pasado y a los actores del mismo, generando un tipo de historia que denuncia y juzga al pasado. Pero trae una problemática, pues al parecer en esta renovación se distorsiona o atrofia la capacidad de hacer historia porque da la idea de que ya está todo zanjado, pues se queda como escrito en la piedra, pues este tipo de historia llega a los colegios, academias y centros de enseñanza mayoritaria y no a la especializada 5 .

Una problemática interesante que plantea es el de tratar de comprender la historiografía a través de las herencias de una generación anterior y del contexto intelectual que va a desarrollar este llamado fin de la historia, y como una generación caracterizada por la historiografía dependentista, además del hecho de que dicha generación proponía una historiografía de una marcada tendencia teórica, que finalmente si es posible decir tuvo algunos alcances importantes o propuestas interesantes, utilizó el método comparativo y

4 Debemos tomar en cuenta que entre los 60 y 80 la formación profesional de historia en el caso de la UNMSM, deja la facultad de Letras y pasa a la nueva facultad de ciencias sociales, tal vez en búsqueda de los nuevos retos, pero sobre todo la influencia de los intelectuales de la generación.

5 Una situación que a partir del siglo XXI está encontrando nuevos brillos más positivos, con una renovación teórica, conceptual y temática de la independencia en este próximo bicentenario que ha sido fruto múltiples debates y nuevos enfoques dejando de lado matices limitantes y aperturando a la historia. 
analógico, para así explicar la realidad histórica peruana en relación con otras realidades. Pero finalmente no provocaron una reacción de identidad, pues hicieron posible una historia con un marcado discurso de juzgar y no comprender, una carga pesada pues la historia de una nueva generación vivía en una situación de limitación política a su vez alimentada por frustraciones lo cual nubla en cierta medida el juicio del historiador, que a su vez acarreó problemas a la próxima generación que a su vez estaba afectada por un contexto mundial y las nuevas tendencias historiográficas; se vio en alguna medida seducida automáticamente a realizar una propuesta intelectual diferente.

En palabras finales del propio autor "Hoy en día toda una tendencia prefiere una historia 'light'. La norma parece ser, esterilizar a la historia. Quitarle todo contenido social, toda proyección, toda discusión teórica, generando un idealismo subjetivo, que conduce a un relativismo limitante a cualquier tipo de generalización en la historia. Se busca lo individual para analizarlo con lujo de detalles, al margen del entorno en que se produce."

Años más tarde, en 1999, Quiroz Chueca, realiza una nueva apreciación historiográfica; toma como ejemplo a la joven historiografía peruana de 1987-1995, mediante el criterio generacional de Macera y la apreciación de Flores Galindo; realiza un ejercicio metodológico de englobar en un arco temporal la producción joven e incluso plantea a través de lo que escriben, textos densos o primeros avances de sus trabajos plasmados en publicaciones en revistas, las fuentes que trabajan y la historia a la cual se están orientando la económica la social, la política, las mentalidades, locales, urbanas, institucionales y de personajes pero el pago al parecer fue un desarrollo heterogéneo de hacer historia, sin tendencias al parecer claras en lo metodológico y teórico, opina aún el hecho de una historia en tono general no reflexiva, de lo pequeño, de las migajas, sin un despejado camino a seguir y a su vez dejar una suerte de continuidad en la medida de la incertidumbre.

Toma en cuenta las influencias de su contexto, el entorno universitario nacional e internacional a través de la salida al extranjero por medio de becas, pero a ello otro elemento, la influencia de los maestros de la universidad. Algo muy interesante que toma en cuenta es que existe una heterogeneidad de tendencias en el desarrollo de la Historia donde existen representantes, afectados por modas, como menciona el autor y otros que continúan con un perfil positivo en el sentido de una continuidad de la Historia en la medida de Historia problema, a pesar de las dificultades y el poco financiamiento existente.

Ante esta opinión no podemos negar qué tipo de historia o historias tan mediáticas constituyeron una respuesta a los años de formación de estos intelectuales en una universidad nacional desorientada y desestabilizada por los años del terror y la sedición que vivió la UNMSM, en donde los intelectuales vivieron dos problemas en carne propia; el descuido institucional y el prejuicio social de su profesión, en donde al parecer lo Light de la historiografía, fue más que una moda una necesidad para superar estos dos problemas en donde la influencia pasajera era un paso lento y atractivo para el desarrollo de la historia,

Para el año 2000 Mirian Laqui realiza una entrevista a dos autoridades de la UNMSM, ambas figuras influyentes en la facultad de ciencias sociales. Ella puntualiza y contextualiza el entorno en el cual se desarrolla la Universidad San Marcos desde los inicios del siglo

6 Quiroz Chueca, Francisco (1995) pág. 148; Los paréntesis son anotaciones al texto que realizo Carlos Lazo en un ejemplo a la revista. 
XXI. Todo ello a partir de una serie de ideas articuladas en un discurso cuyo tenor central es el cambio. Y no solo de siglo, sino también el cambio de tendencias en el desarrollo del centro de producción de la intelectualidad que es la universidad y como sus nuevos timoneles; el director del Post grado de ciencias sociales Manuel Burga y Carlos Lazo director de la EAP de Historia tienen que tomar las riendas del desarrollo de los científicos sociales como herederos de una historia local y mundial tan variante en el quehacer y gestión, y cómo los historiadores viven influenciados por generaciones que se lograron entre de los 60 a los 90; muchos de ellos vivieron la crisis económica y el cambio de modelo, la represión política por parte del Estado, la caída del Muro de Berlín y las confrontaciones ideológicas.

Estos trabajos tópicos han evidenciado a través de su análisis historiográfico, por medio de balances y entrevistas, que las generaciones de historiadores han pasado por diversas coyunturas y los elementos de su estudio son muy diversos y dispersos teniendo como telón de fondo las herencias intelectuales, la labor de la universidad e historiadores y los hechos y cambios, pero también evidencian la carencia de un proyecto, una organicidad institucional, solo por grupos, y por modas y sin capacidad de síntesis o conexión con el presente a pesar de la integración de los estudios con otras áreas.

En esta última apreciación para el 2004 Tubino Arias-Shreiber comenta que existe en la historiografía peruana una ausencia de síntesis porque las temáticas que abordan los historiadores, deben tener una imagen del historiador peruano o perfil a seguir por la intelectualidad joven que se está formando, para él un adelantado a su tiempo a pesar de las limitaciones del mismo y sin continuador en la historiografía es Basadre, un intelectual orgánico que fue capaz de desenvolverse en temas diversos, pero que su interés central era formar una identidad nacional a través de la intelectualidad, para lo que la historia es un baluarte adecuado para hacer posible tal labor, no continuada por los historiadores, el hecho de hacer una historia de la república como idea de formadora de identidad era necesario, pues no solo identificaba a su grupo social, sino que marcaba el inicio de la historia del Perú como nación que si bien fue heredera de una integridad tan compleja como era el mundo andino y su cambio por parte del virreinato era necesaria una historia que formara identidad y la república era el inicio para hacer una historia de síntesis de una pluralidad y un buen inicio. Lo interesante es el análisis en torno al abordaje de una temática por parte de la obra de un autor que se convierta en el perfil del historiador que es de generar un horizonte no solo temático sino de variedad y organicidad y síntesis de situaciones centrales y vitales que la intelectualidad debe tomar en cuenta para comprender a la sociedad a través del tiempo y del espacio.

Estas pocas apreciaciones nos dan una diversidad de ideas o indicadores para poder comprender el desarrollo historiográfico peruano, nos muestran las dificultades para el problema lo cual hace posible vacíos para comprender a los historiadores y la idea de proceso de desarrollo de la historia. Pero sin embargo la dispersión y diversidad son un buen punto de entrada para conocer la evolución historiográfica, la multiplicidad de entradas para conocer y comprender el desarrollo de la historiografía en el Perú; ya sea por medio de generaciones, la producción, las influencias, el tipo de historia que escriben, el grupo al que pertenecen, por medio de una historia presentista, el intelectual y su contexto, 
el grupo social al que pertenece, el tipo de historia que hace o teoría que propone, etc son adecuadas entradas para ver la realización del que hacer histórico peruano.

\section{Un texto clave y la construcción del relato}

Deseo terminar estas páginas tomando en cuenta que dos historiadores realizaron un texto que brinda una opinión generacional sobre el quehacer de la historia en el Perú; a su vez es un instrumento metodológico para recopilar la opinión historiografía mediante una entrevista y se constituye una fuente viva. Hago referencia del texto de Pablo Macera, Conversaciones con Basadre. 2da edición corregida y aumentada con un epílogo y notas de Jorge Basadre. Editado por Mosca Azul editores en 1979.

Macera mediante el desarrollo de este texto trata de reivindicar la utilidad de lo oral como fuente histórica, para comprender el desarrollo de los historiadores utilizando la memoria de los mismos, donde los recuerdos y comprensiones de vida del intelectual nos muestran diversos estilos, coyunturas e investigaciones que forman al historiador, usando a uno de los más icónicos, que influyeron en su generación y en otras generaciones, como es Jorge Basadre. Él utilizará la conversación como medio generador de información, y método por el cual rescatará de la memoria el recuerdo, la crítica, y reflexión de su experiencia de vida, a su vez el dialogo dinámico entre dos intelectuales se puede reconstruir el itinerario de vida y las múltiples circunstancias en las que se desarrolla el individuo.

Es interesante el hecho de cómo Macera toma de Basadre tres sentidos integrados: en el sentido de actor de la historia de una sociedad, como sujeto que hace posible la realización de la Historia y como profesión social, que reconstruye por medio de este diálogo el contexto en el que se desarrolla un intelectual dedicado a la historia en el Perú. Y a su vez Macera se convierte gracias a Basadre en el creador de una fuente directa plasmada en un texto que se puede convertir en modelo metodológico para poder abordar el análisis de la intelectualidad en el Perú.

También es interesante apreciar otro punto cual es la realización de este libro, pues no es solo una conversación trascrita, sino corregida y agregada por los propios protagonistas. A ello hay que agregar que es una segunda edición de este texto y por ende hay algunas correcciones y agregados como en el caso de un epílogo realizado por Basadre.

El libro se puede leer de manera íntegra entendiendo los 6 temas que están íntimamente relacionados, pues desarrollan la temática general que es el intelectual dedicado a la historia en el Perú. Los temas son: el primero el oficio del historiador, en el cual se plantea que la dinámica constante entre el recorrido vital del individuo y el contexto en el que se desarrolla los forja su desempeño en el ejercicio de su profesión. Otro tema, el marxismo y su historia, una mirada a como ha influenciado en el mundo y desarrollado el marxismo y las contradicciones de su aplicación.

Un punto interesante, es el de los historiadores, en el cual trata a dos representantes Riva Agüero y Mariátegui y cómo ellos como intelectuales sus actividades y su contexto afectaron su desarrollo perjudicándolos o silenciándolos; también en este punto trata la relación íntima que debe tener el historiador con la verdad y cómo ella puede ser distorsionada reprimida u ocultada. Pero también es la oportunidad para tocar dos temas en los cuales los historiadores deberían intervenir por ser elementos influyentes en la 
sociedad, el cine y la literatura que es un campo donde Basadre también se desenvolvió como intelectual.

En donde la acción política, es la parte de la vida de un historiador que interviene en la política como una necesidad de clase y muestra la relación intima que antes existía y que en nuestros tiempos no es tan frecuente. Pero también es la oportunidad para conocer y recibir el testimonio de un intelectual y su participación en la política con el APRA y su recorrido por las instituciones públicas dedicadas a la educación. El quinto tema es el punto de cómo los intelectuales se forman en el Perú tomando como ejemplo a Basadre y cual es su función social, lo que se bosqueja a través de la intervención de intelectuales y su participación y respuestas como representantes de grupos diferentes. Esta parte del libro debe ser analizado por el hecho de que intenta evaluar brevemente la participación de grupos intelectuales en el siglo XX del Perú y su participación por el civilismo, pierolismo y el caso de Fernando Casos.

El último tema es un análisis del proceso histórico del Perú y cuál es la visión del historiador maduro y la del discípulo en dirigir el debate de la evolución de la sociedad peruana y cómo el proyecto de nación peruana aún se está estructurando y articulando. El epílogo desarrollado por Basadre nos da una idea de cierre donde el telón de fondo mundial en el cual se desarrolla el Perú a fines de los 70 del siglo XX, es interesante tomar en cuenta para la formación de la intelectualidad en el Perú.

\section{A manera de reflexiones}

Deseo concluir este breve ejercicio introductorio a la apreciación de los historiadores sobre su labor en el devenir de las últimas décadas del siglo XX, expresando que la realización de un ensayo que aborde la formación del historiador es un trabajo muy complicado, el ingreso ha sido posible por la orientación de maestros, quienes me indujeron a hacer un ejercicio de identificación de elementos a través de textos amplios y específicos, el caso de los llamados balances, opiniones y entrevistas identificando condiciones preliminares o vitales que permiten el desarrollo del historiador en el Perú. Debo de agradecer a la Dra. Cristina Florez y al Dr. Fernando Rosas por las charlas de orientación para la realización de este tipo de trabajo preliminar que era más extenso y que decidí recortar, en el cual he tratado de entrelazar las lecturas preliminares y la identificación de condiciones que influyen al historiador en su realización, algo muy importante que debo acotar es el hecho que mientras ingresaba a este tema me permitió recordar cómo influyen la universidad y los docentes en esta labor de forja intelectual. Situación que guardo para otro trabajo de investigación, y a manera de ejercicio de epohistoria pues recordé el apoyo de historiadores que tuve el privilegio de conocer en mi casa de estudios, la Universidad Nacional Mayor de San Marcos, y que constituyen en el tiempo referentes y construcción de redes intelectuales Docentes como; Waldemar Espinoza Soriano, quien me permitió ser su asistente de cátedra, siendo aún estudiante de ciclos superiores, en los cursos de Formación Histórica del Perú y Fuentes del Perú Prehispánico. A un buen docente como Carlos Lazo García, amigo fallecido, que a pesar de las discrepancias u orientaciones políticas fue un gran amigo que me enseñó que la limitación del tiempo y las discrepancias políticas no son obstáculo para orientar, pues no solo me orientaba en el desarrollo de investigaciones en crónicas y su 
discurso, a su vez me permitió ser asistente de cátedra en el curso de Filosofía de la Historia con el tema de la historia cultural, gracias a él pude conocer a la Dra. Cristina Florez Dávila, una persona muy importante, mi gran maestra y orientadora, a quien aprecio mucho y valoro sus consejos que son parte de mi vida. A los profesores Jóvenes en aquel entonces de fines de los 90 e inicios del nuevo milenio, Cesar Puerta, Francisco Quiroz Chueca, David Rodriguez, Raul Adanaque, Carlos Carcelen y Carlota Casalino, que fueron desde sus cursos docentes motivadores, inquietantes, iconoclastas, proyectistas, metódicos y dinámicos con los cuales el debate y los posibles alcances u orientaciones siempre fueron interesantes. Una mención especial es al Profesor Cristóbal Aljovín, quien siempre tenía tiempo para poder dar un consejo e incluso apoyo para la realización de algún evento académico en apoyo de los estudiantes de historia. Un agradecimiento en comprender y apreciar la paleografía y la archivística se los debo a no solo la experiencia laboral o la investigación, sino a la paciencia de Camilo Vicente y Ruth Borja que eran considerados como docentes de una actividad técnica y práctica que en el momento de búsqueda de información se convierte en un arma vital.

Y finalizo, agradeciendo en estas líneas, pues no creo, que estaría escribiendo sobre historia sino fuese no solo por los padres intelectuales, pues desde hace algunos años al final de cada semestre y en las aulas de la universidad les dedico a mis estudiantes unas palabras, "... ser Historiador en nuestro país es una gran profesión, que me ha dado lo mejor de mi vida, construir y hacer crecer mi familia; la historia me ha brindado puertas para la búsqueda de una comprensión sobre la historia de nuestro país y me ha otorgado la oportunidad de compartirla en la labor tan humana que es ser docente en una universidad...." Debo acotar que soy Historiador por el Apoyo de mi madre Alejandrina que se alegró cuando ingresé a San Marcos para ser lo que deseaba a pesar de haber renunciado a otra universidad y otra carrera profesional. Mi fallecido tío abuelo Alejandro en el cual pude encontrar a esa figura artística de la familia preceptora y protectora que me motivaba a vivir y sentir lo que hacía y por la familia en donde todo sacrificio es en pro de un fin mayor. Mi hermano Jorge que me motivó para reingresar a la universidad y dejar de lado la carrera técnica de la Marina de Guerra del Perú, sus consejos, aunque duros sobre la universidad y la vida universitaria siempre fueron bien acogidos. Mi querida compañera y esposa Carmen de quien vivo enamorado y ella se enamoró de mí en las aulas de la universidad y decidió acompañarme en el camino de ser novios, esposos, compañeros y padres. En donde los consejos, la paciencia, la crítica y el apoyo a nuestras profesiones son siempre nuestra medicina a pesar de las diversas adversidades. A mis estudiantes y que hoy son profesionales, Joel, Pilar, José, (estudiantes de la UNMSM) Santiago, Jennifer, Sandro, Edgar, entre otros (quienes son aun estudiantes de la URP) que en sus visitas o encuentros eventuales me cuentan con gran entusiasmo su vida y sus experiencias en el ámbito profesional. 


\section{BIBLIOGRAFÍA}

Basadre, J. (1981). La vida y la historia. Ensayos sobre personas, lugares y problemas. Segunda edición revisada y aumentada por el autor. Lima: Taller industrial.

Basadre, J. (1978). "Docencia Histórica" En: Apertura. Textos sobre temas de historia, educación, cultura y política, escritos entre 1924 y 1977. (Selección, edición, prólogo y notas introductorias de Patricio Ricketts. Lima: Ediciones taller. 91-182.

Burga, M. (2005). La Historia y los Historiadores en el Perú. UNMSM-Universidad Inca Garcilaso de la Vega. Lima: 231.

Flores Galindo, A. (1988). "La imagen y el espejo: la historiografía peruana 1910-1986." En: Márgenes encuentro y debate. Año II, № 4. Lima: Casa de estudios del socialismo SUR. 55-83.

Glave, L. M. (1999). “El objeto de la historia en el Perú". En: Diálogos en Historia. № 1 Historia colonial e historiografía peruana. Grupo de estudios e investigaciones Clío - UNMSM. Lima. Págs. 95 - 107.

Kapsoli E., W. (2001). (Comp.) Historia e Historiadores. Lima: Universidad Ricardo Palma.

Laqui, M. (2000) "San Marcos, ciencias sociales e Historia: un balance y dos entrevistas." En: Perú contemporáneo. Lima. 41-47.

Lazo García, C. (2005). "Historia e historiadores sociales en la historia del Perú del siglo XX." Praxis en la Historia. Lima: Taller de estudios filosóficos. III, (4). Lima. 13-27.

Lituna P., L. (1973). "Cacisiaco y el dialogo 'contra los académicos.' Humanidades No 5 PUCP. Lima: PUCP. 7-14.

Macera, P. (1979). Conversaciones con Basadre. 2da edición corregida y aumentada con un epilogo y notas de Jorge Basadre. Lima: Mosca Azul.

(1988). "Historia en el Perú ciencia e ideología." En: Trabajos de Historia. Lima: Tomo I. G. Herrera Editores - Facultad de Ciencias Sociales UNMSM. 3-20

Miró Quesada, F. (1978). "Historia y teoría en la obra de Jorge Basadre." En: Historia problema y promesa, homenaje a Jorge Basadre. Lima: PUCP. I, 191-206.

Nuñez, E. (1984). "Raúl Porras y los nuevos senderos de la historia peruana." En: Homenaje a Raúl Porras Barrenechea. Lima: UNMSM-Instituto Raúl Porras Barrenechea-Centro de altos estudios e investigaciones peruanas. 73-79.

Puente Candamo, J, A. de la. (1987). "Reflexiones sobre la obra historiográfica de Aurelio Miró Quesada Sosa." En: Libro de homenaje a Aurelio Miro Quesada Sosa. Tomo I. Lima: Talleres Gráficos P. L. Villanueva S. A. Editores. 133-140.

Quiroz Chueca, F. (1995). "Historiografía dependentista y el fin de la historia." En: Nueva Síntesis. Revista de Humanidades. Año II (3). Lima.

(1999).“La Historiografía joven peruana 1987-1995.”En:Diálogos en Historia. Historia colonial e historiografía peruana. Lima: Grupo de estudios e investigaciones Clío- UNMSM. 1, 109-128.

Ragas Rojas, J. F. (1999). "La juventud de Riva Agüero y Mariátegui, comentario a dos tesis de la PUCP." En: Diálogos en Historia. Historia colonial e historiografía peruana. Lima: Grupo de estudios e investigaciones Clío - UNMSM. 1, 200-202 
(2004) "Los Annales en el Perú. Basadre, Febvre y Braudel en perspectiva (1929-1953)." En: Historia y Cultura Lima: INC - PUCP. 25, 203-218

Rivera Serna, R. (1984). "Raúl Porras, Historiador." En: Homenaje a Raúl Porras Barrenechea. Lima: UNMSM-Instituto Raúl Porras Barrenechea-Centro de altos estudios e investigaciones peruanas. 191-205

Tubino Arias-Schreiber (2004). "Perú: la ausencia de síntesis." En: Historia y Cultura No25. Lima: INC - PUCP. 59-74

Zea, L. (1978). "La filosofía de la historia Latinoamericana” En: Historia problema y promesa, homenaje a Jorge Basadre. Tomo I. Lima: Fondo editorial PUCP. 341-352 